\title{
The Anti-Angiogenic Agent Lenvatinib Induces Tumor Vessel Normalization and Enhances Radiosensitivity in Hepatocellular Tumors
}

Norikazu Une

Tohoku University: Tohoku Daigaku

Mayumi Takano-Kasuya

Tohoku University: Tohoku Daigaku

Narufumi Kitamura

Tohoku University: Tohoku Daigaku

Mineto Ohta

Tohoku University: Tohoku Daigaku

Tomoya Inose

Tohoku University: Tohoku Daigaku

Chihiro Kato

Tohoku University: Tohoku Daigaku

Ryuichi Nishimura

Tohoku University: Tohoku Daigaku

Hiroshi Tada

Tohoku University: Tohoku Daigaku

Shigehito Miyagi

Tohoku University: Tohoku Daigaku

Takanori Ishida

Tohoku University: Tohoku Daigaku

Michiaki Unno

Tohoku University: Tohoku Daigaku

Takashi Kamei

Tohoku University: Tohoku Daigaku

Kohsuke Gonda ( $\nabla$ gonda@med.tohoku.ac.jp )

Tohoku University https://orcid.org/0000-0002-0564-6033

\section{Research Article}

Keywords: Lenvatinib, Hepatocellular carcinoma, Vascular normalization, Sorafenib, Radiation therapy, Computed tomography 
Posted Date: February 16th, 2021

DOI: https://doi.org/10.21203/rs.3.rs-194550/v1

License: (9) This work is licensed under a Creative Commons Attribution 4.0 International License. Read Full License

Version of Record: A version of this preprint was published at Medical Oncology on April 21st, 2021. See the published version at https://doi.org/10.1007/s12032-021-01503-z. 


\section{Abstract}

The evaluation of angiogenesis inhibitors requires the analysis of the precise structure and function of tumor vessels. The anti-angiogenic agents lenvatinib and sorafenib are multi-target tyrosine kinase inhibitors that have been approved for the treatment of hepatocellular carcinoma (HCC). However, the different effects on tumor vasculature between lenvatinib and sorafenib are not well understood. In this study we analyzed the effects of both drugs on vascular structure and function, including vascular normalization, and investigated whether the normalization had a positive effect on a combination therapy with the drugs and radiation using micro X-ray computed tomography with gold nanoparticles as a contrast agent, as well as immunohistochemical analysis and interstitial fluid pressure (IFP) measurement. In mice subcutaneously transplanted with mouse HCC cells, treatment with lenvatinib or sorafenib for 14 days inhibited tumor growth and reduced the tumor vessel volume density. However, analysis of integrated data on vessel density, rates of pericyte-covering and perfused vessels, tumor hypoxia, and IFP measured 4 days after drug treatment showed that treatment with $3 \mathrm{mg} / \mathrm{kg}$ of lenvatinib significantly reduced the microvessel density and normalized tumor vessels compared to treatment with $50 \mathrm{mg} / \mathrm{kg}$ of sorafenib. These results showed that lenvatinib induced vascular normalization and improved the intratumoral microenvironment in HCC tumors earlier and more effectively than sorafenib. Moreover, such changes increased the radiosensitivity of tumors and enhanced the effect of lenvatinib and radiation combination therapy, suggesting that this combination therapy is a powerful potential application against HCC.

\section{Introduction}

Angiogenesis is essential for solid tumors to grow beyond 2-3 $\mathrm{mm}^{3}$, and newly formed tumor vessels are closely involved in further tumor growth and metastasis [1,2]. Tumor cells release angiogenic factors such as vascular endothelial growth factor (VEGF), platelet-derived growth factor (PDGF), and fibroblast growth factor (FGF), which act on nearby blood vessels to cause tumor angiogenesis [3, 4]. These angiogenesis-promoting factors lead to the rapid development of tumor neovascular vessels that are irregularly shaped, dilated, leaky, and fragile [5,6]. Approximately 50 years ago, Folkman et al. suggested that angiogenesis is necessary for tumor growth and that angiogenesis inhibitors have anti-tumor effects via the shrinkage and disruption of tumor blood vessels, followed by the starving of oxygen and nutrient supply to cancer cells [7]. Angiogenesis inhibitors have been theorized to induce hypoperfusion in tumor tissue, thereby leading to hypoxia in these tissues $[1,7]$. Thus, hypoperfusion may aggravate drug delivery through vessels and cause drug resistance; moreover, inhibitor-induced hypoxia may reduce the effect of radiotherapy on tumors $[8,9]$.

However, a new idea has recently been proposed, in which angiogenesis inhibitors remodel abnormal tumor vessels into normal vessels, referred to as "tumor vascular normalization" $[5,10]$. Normalization improves the tumor microenvironment and inhibits tumor growth and metastasis [6]. In addition, normalization of tumor vessels also improves blood perfusion and tumor oxygenation in tumor tissues, which may enhance the efficacy of chemotherapy and radiation therapy [11, 12]. These new ideas differ 
from the previous concept. Angiogenesis inhibitors have recently been shown to improve the efficacy of immune checkpoint inhibitor (ICI) therapy $[13,14]$. Therefore, appropriate evaluation and validation of the effects of angiogenesis inhibitors on tumor vessel normalization are important for the development of drugs for $\mathrm{ICl}$ therapy. Moreover, tumor oxygenation via normalization may also increase tumor radiosensitivity, which is important for the effects of radiation therapy [12].

Hepatocellular carcinoma (HCC) is the third leading cause of cancer-related deaths worldwide [15]. Liver resection or radiofrequency ablation (RFA) are the only curative therapies for HCC [16]. However, these treatments are not always selected because more than half of HCC patients are diagnosed at an advanced stage of disease [17]. Although advanced HCC patients who are unsuitable for resection or RFA are treated with transcatheter arterial chemoembolization, radiotherapy, or chemotherapy, their prognosis remains poor [18].

Sorafenib, an anti-angiogenic agent and multi-target tyrosine kinase inhibitor (TKI) directly acts and inhibits angiogenesis factor receptors, such as vascular endothelial growth factor receptor (VEGFR) and platelet-derived growth factor receptor (PDGFR), and was approved in Japan for the treatment of HCC in $2009[19,20]$. According to the results of a clinical trial, sorafenib prolonged overall survival (OS) by 3 months compared to the placebo group; however, the objective response rate (ORR) was only $2.3 \%$ [19]. In contrast, lenvatinib, a novel multi-target TKI with anti-angiogenesis activity, was approved in Japan for the treatment of HCC in 2018. In another clinical trial, lenvatinib was non-inferior to sorafenib in the primary outcome of OS and improved the ORR compared to that of sorafenib (40.6\% vs. 12.4\%) [21]. In Japan, sorafenib and lenvatinib are approved as first-line treatments for HCC, while regorafenib and ramucirumab are approved as second-line treatments for sorafenib-refractory HCC [22, 23]. Since both sorafenib and lenvatinib are multi-target TKIs, they have different abilities to inhibit VEGFR, PDGFR, and fibroblast growth factor receptors (FGFR); in particular, the inhibitory effect of lenvatinib on VEGFR is higher than that of sorafenib $[24,25]$. However, the varied effects of lenvatinib and sorafenib on tumor vasculature in HCC are not well understood. In fact, previous studies could not clearly detect differences in the effects of lenvatinib and sorafenib on the microvascular structure and tumor microenvironment.

Antitumor effects of angiogenesis inhibitors have often been evaluated using immunohistochemical staining with anti-CD31, CD34, or von-Willebrand-Factor antibodies as target markers in vascular endothelial cells [26]. In breast and prostate cancer, high microvessel density (MVD) in tumor tissue is considered a poor prognostic factor $[27,28]$. Therefore, the evaluation of intratumoral vasculature is an important factor that correlates with therapeutic effects and patient prognosis. However, there is a limitation to the evaluation of MVD in tumor tissue because the evaluation area is a small part of a huge tumor and there is heterogeneity in tumor blood vessels $[29,30]$. Thus, the accurate assessment of the effect of angiogenesis inhibitors on tumor vessels requires an evaluation of the entire tumor vasculature in addition to the investigation of tumor tissue sections.

We previously performed three-dimensional (3D) in vivo analysis of whole-tumor vascular structures in tumor-bearing mice using $15 \mathrm{~nm}$ gold nanoparticles supported with polyethylene glycol chains (AuNPs) 
as a contrast agent for micro X-ray computed tomography ( $\mu$ X-ray CT) [31]. In this study, we evaluated the effects of lenvatinib and sorafenib on tumor growth and the volume density of vessels measuring $50 \mu \mathrm{m}$ or more in whole tumors using $\mu \mathrm{X}$-ray CT and on the area density of microvessels on tissue sections, percentage of vessels covered with pericytes, rate of perfused vessels, tumor hypoxia, and interstitial fluid pressure (IFP) using immunohistochemical analysis or IFP measurement. Additionally, we demonstrated the synergetic effects of lenvatinib and radiation therapy using $\mu \mathrm{X}$-ray $\mathrm{CT}$ and immunohistochemistry although the synergetic effects were not shown in previous studies. Our results showed that lenvatinib induced vascular normalization and improved the intratumoral microenvironment more effectively than sorafenib and that such changes enhanced the effects of lenvatinib and radiation combination therapy.

\section{Methods}

\section{Experimental animals}

Female BALB/c nude mice aged 5-6 weeks were purchased from Charles River Laboratories, Japan. All animals were treated in accordance with the guidelines approved by the Committee on Animal Experiments at Tohoku University. All surgical processes were performed on mice under anesthesia with the subcutaneous injection of a combination of $0.3 \mathrm{mg} / \mathrm{kg}$ of medetomidine, $4.0 \mathrm{mg} / \mathrm{kg}$ of midazolam, and $5.0 \mathrm{mg} / \mathrm{kg}$ of butorphanol.

\section{Cell culture and drugs}

Hepa1-6 cells, derived from murine hepatoma, were purchased from RIKEN BRC, Japan. The cells were cultured in Dulbecco's minimum essential medium (DMEM) containing $10 \%$ fetal bovine serum and incubated at $37^{\circ} \mathrm{C}$ in a mixture of $5 \% \mathrm{CO}_{2}$.

Lenvatinib was obtained from Chemscene (Monmouth Junction, $\mathrm{NJ}$ ), and sorafenib was obtained from Toronto Research Chemicals (North York, Canada). Lenvatinib was dissolved in $0.5 \mathrm{w} / \mathrm{v} \%$ methylcellulose for in vivo studies and in dimethyl sulfoxide (DMSO) for the in vitro studies. Sorafenib was dissolved in DMSO for both in vivo and in vitro studies.

\section{Cell experiments in vitro}

Hepa1-6 cells were seeded at a density of $5 \times 10^{4}$ cells in $35-\mathrm{mm}$ well plates and were treated the next day with various concentrations of lenvatinib $(1-30 \mu \mathrm{M}$; dissolved in $0.1 \% \mathrm{DMSO})$, sorafenib $(0.3-30 \mu \mathrm{M}$; dissolved in $0.1 \%$ DMSO), or control medium (0.1\% DMSO). After $24 \mathrm{~h}, 48 \mathrm{~h}$, and $72 \mathrm{~h}$, the cells were counted under a microscope $(n=3)$.

\section{Preparation of the tumor-bearing mouse models}

Hepa1-6 cells $\left(1 \times 10^{7}\right)$ suspended in DMEM were subcutaneously transplanted into the right flanks of mice. Approximately seven days after the injection, tumor-bearing mice with tumor sizes of 150-200 $\mathrm{mm}^{3}$ were randomly assigned to one of five groups: control group (orally treated with saline), sorafenib 
groups (30 mg/kg and $50 \mathrm{mg} / \mathrm{kg}$, dissolved in 10\% DMSO), or lenvatinib groups ( $3 \mathrm{mg} / \mathrm{kg}$ and $10 \mathrm{mg} / \mathrm{kg}$, dissolved in methylcellulose). Saline, sorafenib, and lenvatinib were administered via oral gavage once daily (Fig. 1a). The tumor dimensions were measured using a caliper every alternate day and the tumor volume was calculated using the following formula:

Tumor volume $=1 / 2 \times($ long axis $) \times(\text { short axis })^{2}$.

Day 1 was the first day of drug administration and the mice were treated with saline, sorafenib, or lenvatinib until day 4 or day 14 . On day 4 or day 15 , the mice were anesthetized and injected intravenously with AuNPs, followed by $\mu$ X-ray CT imaging. After the CT imaging, the mice were euthanized, and tumors were harvested and fixed.

To administer lenvatinib and radiation combination therapy, tumor-bearing mice (tumor size 150-200 $\mathrm{mm}^{3}$ ) were randomly assigned to one of four groups: control group (with saline and without irradiation), irradiation group (with saline and with 6 Gy irradiation on day 4), lenvatinib group (with $3 \mathrm{mg} / \mathrm{kg}$ lenvatinib and without irradiation), and combination group (with $3 \mathrm{mg} / \mathrm{kg}$ lenvatinib and $6 \mathrm{~Gy}$ irradiation on day 4). On day 15 , the mice were scanned using $\mu$ X-ray CT and their tumors were harvested after euthanasia.

\section{Fabrication of AuNPs as a contrast agent}

The AuNPs were synthesized according to the method described in our previous study [31]. Briefly, 99.4 mg of $\mathrm{HAuCl}_{4}$ (FUJIFILM Wako Pure Chemical Corporation, Osaka, Japan, 99\%) was dissolved in $233 \mathrm{~mL}$ ultrapure water and heated to a boil. The $\mathrm{HAuCl}_{4}$ solution was vigorously stirred, and $28 \mathrm{~mL}$ of $39 \mathrm{mM}$ sodium citrate was added to the solution under constant stirring. The sample was boiled for $30 \mathrm{~min}$. After cooling the sample solution to $25^{\circ} \mathrm{C}, 99.4 \mathrm{mg}$ of HS-PEG-COOH (thiol carboxylic polyethylene glycol (PEG), MW 5000, Nanocos Inc., New York, NY) was added to the sample and incubated at $25^{\circ} \mathrm{C}$ for $12 \mathrm{~h}$, with constant stirring to modify PEG chains on the surface of AuNPs.

\section{$\mu \mathrm{X}$-ray CT}

$\mu \mathrm{X}$-ray CT imaging was performed using a $\mu \mathrm{CT}$ scanner (SkyScan1176, Bruker, Billerica MA). The CT system can minimize motion artifacts when scanning live mice administered inhalation anesthesia with isoflurane. CT images were acquired at $50 \mathrm{kVp}$ and $490 \mu \mathrm{A}$. The CT image size was $18 \times 18 \mu \mathrm{m}$ and the slice thickness was $18 \mu \mathrm{m}$. An aluminum $0.5-\mathrm{mm}$ compensating filter was used. To evaluate enhancement by the contrast agent, the quantification of CT signals was expressed in Hounsfield units (HUs). The CT data were analyzed based on the HUs in a region of interest.

\section{$\mu \mathrm{X}$-ray CT imaging with AuNPs}

Tumor-bearing mice were anesthetized and injected intravenously with $200 \mu \mathrm{L}$ of $1.0 \mathrm{M} 15 \mathrm{~nm}$ AuNPs. CT scanning was then performed. The acquired CT data were reconstructed using NRecon software (Bruker) and displayed in 3D using CTvox software (Bruker). The 3D blood vessel images were displayed using 
maximum intensity projection. The tumor area was manually set to the regions of interest using CTAn software (Bruker) and tumor and vessel volumes were calculated. The tumor blood vessel volume was calculated by considering regions with CT values of $\geq 570-600 \mathrm{HU}$ to indicate a blood vessel as tumor CT values scanned with plain CT were $80-100 \mathrm{HU}$ and voxels over $570-600 \mathrm{HU}$ comprised only $0.1 \%$ of all tumor voxels. In this protocol, the CT images were visualized and analyzed in vessels measuring over $50 \mu \mathrm{m}$.

\section{Immunohistochemical analyses}

Mouse tissue samples were immediately frozen in Tissue-Tek ${ }^{\circledR}$ O.C.T. compound (SAKURA, Tokyo, Japan) or fixed in $10 \%$ formalin and prepared in paraffin. OCT compound-embedded frozen samples were cut into 15- $\mu \mathrm{m}$ thick sections while paraffin-embedded samples were cut into 3- $\mu \mathrm{m}$ thick sections. Immunofluorescence analysis was performed to evaluate the area densities of the tumor microvessels, vascular normalization, and tumor hypoxia. The frozen tissue sections were fixed in $5 \%$ paraformaldehyde for $15 \mathrm{~min}$ and the paraffin-embedded tissue sections were deparaffinized in xylene and hydrated with a graded alcohol series and distilled water. These tissue slides were blocked using $5 \%$ goat or donkey serum for $1 \mathrm{~h}$. The sections were then incubated with the following primary antibodies: anti-CD31 (1:200, Angio-Protemie, Boston, MA), anti-a-smooth muscle actin (SMA) (1:100, Abcam, Cambridge, UK), or anti-pimonidazole (1:50, Hypoxyprobe Inc., Burlington, MA) antibody overnight at $4{ }^{\circ} \mathrm{C}$. The tissues were subsequently washed with phosphate-buffered saline (PBS) and incubated for $1 \mathrm{~h}$ at $25^{\circ} \mathrm{C}$ with a second antibody (diluted 1:400) conjugated to AF488, AF568, or Cy5 (Abcam). The cell nuclei were counterstained with 4', 6-diamidino-2-phenylindole (DAPI). The slides were observed under BZ-X800 fluorescence (KEYENCE, Osaka, Japan) or laser confocal LSM 780 (Zeiss, Oberkochen, Germany) microscopes.

\section{Histological analysis}

Tumor vessel perfusion was quantified on tumor cryosections following intravenous injection with $50 \mu \mathrm{g}$ of AF649-labeled Lycopersicon esculentum lectin (Vector Laboratories, Burlingame, CA) into tumorbearing mice. The area densities of the tumor microvessels, percentages of vessels covered with pericytes (a-SMA and CD-31 colocalization area/CD-31 localization area), and percentages of perfused vessels (lectin positive area/CD-31 positive area) were captured in five fields at 20x magnification. Tumor hypoxia was determined using pimonidazole. Pimonidazole $(60 \mathrm{mg} / \mathrm{kg})$ was injected intraperitoneally and the tumor was harvested after $60 \mathrm{~min}$. The tissue sections were analyzed by immunofluorescence with anti-pimonidazole antibody (1:100). All immunofluorescence images were analyzed using ImageJ software.

Hematoxylin and Eosin (H\&E) staining was performed to evaluate tumor necrosis and organ dysfunction. The H\&E-stained slides covered the entire tissue section at 20x magnification. For quantification of the necrotic area, all images were stitched using a BZ-X800 microscope to obtain an image of the entire 
tissue section. The percentage of hematoxylin-stained tissue was defined as the viable area. The necrotic areas were calculated using ImageJ software.

To evaluate numbers of microvessels and Ki-67-positive cells, paraffin-embedded tissues were deparaffinized and antigen retrieval was performed in $10 \mathrm{mM}$ sodium citrate buffer for 5 min in a $121^{\circ} \mathrm{C}$ autoclave. After antigen retrieval, $5 \%$ goat serum was used for blocking at $25^{\circ} \mathrm{C}$ for $1 \mathrm{~h}$ and the sections were incubated with primary antibodies (CD31, 1:50; Ki-67, 1:100) (Abcam) overnight at $4{ }^{\circ} \mathrm{C}$. The sections were then stained with 3,3'- diaminobenzidine tetrahydrochloride (DAB) and counterstained with hematoxylin.

MVD was determined as previously described [27]. Briefly, two researchers independently calculated the MVD. Microvessels were defined as vascular regions surrounded by CD31-positive endothelial cells or cell clusters immunostained with anti-CD31 antibody and DAB, which were clearly separated from the surrounding tumor and stromal cells. The sections were screened at a lower magnification (10x) to identify five vascularized areas. Within the selected areas, microvessels were counted under high magnification (40x). MVD was the average number of microvessels in the five fields.

$\mathrm{Ki}-67$ was quantified using a method similar to that described above for the MVD analysis. The sectioned samples were screened at lower magnifications to identify the representative areas. The numbers of DABpositive nuclei were counted and compared to the total numbers of nuclei under high magnification. Five fields were calculated for each tumor and the percentage of Ki-67-positive cells was the average number from five fields.

\section{IFP measurement with the wick-in-needle technique}

IFP of the tumor was measured in the tumor-bearing mice using the wick-in-needle technique after lenvatinib, sorafenib, or control treatment [32,33]. Briefly, a 23-gauge needle filled with multifilamentous nylon thread was connected to a transducer and amplifier (ADInstruments, Dunedin, New Zealand). The tumor-bearing mice were anesthetized and fixed in a position. The needle was then inserted into the HCC tumor $2 \mathrm{~mm}$ from the surface and left in place until the measured pressure had stabilized. The IFP was measured in at least two different tumor regions.

\section{X-ray irradiation}

X-ray irradiation was performed using a device to administer a dose of 6 Gy (80 kV, 1.25 mA, MX-80Labo, mediXtec Japan (Chiba, Japan).

\section{Statistical analyses}

JMP software (SAS institute, Cary NC) was used to perform general statistical analyses. All measurements are expressed as means \pm the standard error of the mean (SEM). Student's t-tests and one-way analysis of variance test, followed by Tukey multiple comparison test were used for comparisons between two groups and multiple groups. $P \otimes 0.05$ was considered statistically significant. 


\section{Results}

\section{Effects of lenvatinib and sorafenib on tumor growth and tumor vessel density}

To investigate the effect of lenvatinib and sorafenib on tumor progression, saline, $30 \mathrm{mg} / \mathrm{kg}$ or $50 \mathrm{mg} / \mathrm{kg}$ sorafenib, or $3 \mathrm{mg} / \mathrm{kg}$ or $10 \mathrm{mg} / \mathrm{kg}$ lenvatinib were administrated to tumor-bearing mice once daily for 14 days after the tumor volume had reached $150-200 \mathrm{~mm}^{3}$. The tumor dimensions were measured using a caliper every alternate day (Fig. 1a). Compared to the control mice, mice treated with lenvatinib (3 mg/kg or $10 \mathrm{mg} / \mathrm{kg}$ ) and sorafenib (30 mg/kg or $50 \mathrm{mg} / \mathrm{kg}$ ) showed significantly inhibited tumor growth at day 14 (Fig. 1b). However, cell proliferation following treatment with sorafenib for $72 \mathrm{~h}$ was more potently inhibited at the same concentration as that with lenvatinib (Fig. S1), indicating that the anti-tumor effects of lenvatinib and sorafenib differed in vitro and in vivo.

Next, we examined tumor volume and intratumoral vessels on day 15 using $\mu$ X-ray CT imaging with AuNPs. $\mu$ X-ray CT imaging enables the visualization of tumor vessels of approximately $50 \mu \mathrm{m}$ or more. Fig. 1c shows the 3D micromorphology of untreated tumor vessels and those treated with angiogenesis inhibitors. Analysis of the CT imaging data showed significantly decreased tumor volume, vessel volume, and vessel volume density (vessel volume/tumor volume) in all groups treated with lenvatinib or sorafenib compared to those in the control group (Fig. 1d). The tumor volumes calculated using $\mu \mathrm{X}$-ray CT and caliper were similar. Additionally, the vessel volume in the $10 \mathrm{mg} / \mathrm{kg}$ lenvatinib treatment group was significantly lower than those in the $30 \mathrm{mg} / \mathrm{kg}$ and $50 \mathrm{mg} / \mathrm{kg}$ sorafenib treatment groups. While vascular volume density treated with $10 \mathrm{mg} / \mathrm{kg}$ of lenvatinib tended to be decreased compared to sorafenib treatment, no significant difference in the volume densities of the intratumor vessels were observed between the groups treated with lenvatinib and sorafenib.

\section{Lenvatinib induces vascular normalization earlier than sorafenib}

Previous studies could not clearly detect differences in the effects of lenvatinib and sorafenib on the microvascular structure and tumor microenvironment. The determination of the mechanisms of action of both drugs at which the differences in drug efficacy to the microvascular structure and tumor microenvironment first occur may inform drug discovery. These changes in microvascular structure and tumor microenvironment may induce tumor vessel normalization and enhance the effects of ICI therapy and radiotherapy, which are promoted via improvements in the microenvironment by the normalization, and, thus, contribute to the development and application of angiogenesis inhibitors. Since the differences in the initial reaction are too large between drug conditions in which differences are clearly observed, this study instead analyzed the differences in the initial changes between two drugs with similar antitumor effects on tumor growth. Thus, we focused on treatment with $50 \mathrm{mg} / \mathrm{kg}$ of sorafenib and $3 \mathrm{mg} / \mathrm{kg}$ of lenvatinib. As shown in the graph in Fig. 1b, the difference between the two drug groups first began to appear on day 4 . Thus, this study focused on these conditions.

After tumor volume reached $150-200 \mathrm{~mm}^{3}$, the tumors were treated with saline (control), $50 \mathrm{mg} / \mathrm{kg}$ sorafenib, or $3 \mathrm{mg} / \mathrm{kg}$ lenvatinib. The tumor sizes were measured using caliper on days 0,2 , and 4 (Fig. 
$2 a$ and $b$ ). To understand the differences between both drugs in detail, we imaged the intratumoral vasculature using $\mu$ X-ray CT with AuNPs (Fig. 2c). The volume densities of the tumor vessels calculated via CT did not differ significantly between the three groups on day 4 (Fig. 2d). Although $\mu$ X-ray CT with AuNPs in this study can be used to visualize vessels measuring $50 \mu \mathrm{m}$ or more, this technique cannot easily visualize blood vessels that are smaller than $50 \mu \mathrm{m}$ and vascular-related tumor microenvironments. Therefore, to investigate the effect of lenvatinib and sorafenib on microvessel structures measuring $50 \mu \mathrm{m}$ or less, the percentages of vessels covered with pericytes, and the percentages of perfused vessels were assessed using immunohistochemical analysis with anti-CD31 antibody, which is a marker of endothelial cells in both mature and immature vessels. Immunohistochemical analysis with anti-CD31 antibody and DAB, which is a conventional method of evaluating vessel number $[29,30]$, showed significantly smaller numbers of vessels in the lenvatinib and sorafenib treatment groups compared to that number in the control group (Fig. S2). However, no significant difference was observed between lenvatinib and sorafenib treatments (Fig. S2). Therefore, we next measured the area densities of vessels using a more sensitive method; namely, immunofluorescence with anti-CD31 antibody. The results showed significantly decreased CD31-positive area densities in the group treated with lenvatinib compared to those in the sorafenib and control groups (Fig. 2e and f), suggesting that lenvatinib might remodel tumor microvessels thinner and shorter than sorafenib because there was no difference in the number of vessels between lenvatinib and sorafenib treatment.

Next, we evaluated the percentages of vessels covered with pericytes (defined as the a-SMA and CD31 colocalization area/CD-31 localization area) by double immunostaining with anti-CD31 and anti-a-SMA, a pericyte marker. Pericyte coverage is an indicator of tumor vascular normalization [10]. The immunostaining results showed a significantly higher percentage in the group treated with lenvatinib than that in the control group (Fig. 2e and g). There was no significant difference in the percentages of pericyte-covering vessels between the sorafenib and control groups (Fig. $2 \mathrm{~g}$ ).

We then examined the percentages of perfused vessels. First, fluorescence-labeled lectin, which interacts with the vascular endothelium, was intravenously injected into mice and perfused vessels were labeled within minutes [34]. To calculate the percentages of perfused vessels (defined as lectin and CD31 colocalization area/CD-31 localization area) in the tumors, we stained lectin-labeled tumor sections with anti-CD31 antibody. The results showed a significantly higher percentage in the group treated with lenvatinib those in the group treated with sorafenib and the control group (Fig. $2 \mathrm{~h}$ and i). We observed no significant difference in percentages between the sorafenib and control groups (Fig. 2i).

\section{Vascular normalization induced by lenvatinib reduces hypoxia and IFP in tumors}

The observed increase in the percentage of pericyte coverage and perfused vessels in the group treated with lenvatinib suggested that lenvatinib induced tumor vessel normalization earlier than sorafenib. Therefore, we next investigated the effect of normalization on hypoxia and IFP in tumors. The groups treated with saline, $50 \mathrm{mg} / \mathrm{kg}$ sorafenib, or $3 \mathrm{mg} / \mathrm{kg}$ lenvatinib were intraperitoneally injected with pimonidazole, a marker for tumor hypoxia, on day 4 . After $60 \mathrm{~min}$, the tumors were harvested and the 
cross-sections were analyzed via immunofluorescence with anti-pimonidazole antibody (Fig. 3a). The percentages of pimonidazole-positive areas among total viable areas were significantly lower in the groups receiving lenvatinib or sorafenib treatment (Fig. 3b). We observed no significant difference in pimonidazole-positive areas between the lenvatinib and sorafenib treatment groups (Fig. 3b).

Next, to evaluate the IFP in tumors after treatment with lenvatinib and sorafenib, drug-treated mice were anesthetized and the IFP was measured using the wick-in-needle technique [32]. The IFP in the group treated with lenvatinib was significantly lower than those in the sorafenib and control groups (Fig. 3c). However, we observed no significant difference in IFP between the sorafenib and control groups (Fig. 3c). These results indicated that the tumor vessel normalization induced by lenvatinib improved the tumor microenvironment.

\section{Vascular normalization induced by lenvatinib enhances the synergistic effect of lenvatinib and radiation combination therapy in HCC tumors}

As hypoxic tissues show resistance to radiation [35], we hypothesized that lenvatinib-induced normalization of tumor vessels and improvement of tumor microenvironment increased the antitumor effects of radiation. In the previous studies, synergetic effects of lenvatinib and radiation therapy in HCC tumors were not shown. The treatment protocol for lenvatinib and radiation combination therapy is shown in Fig. 4a. A single 6-Gy X-ray irradiation dose was administered on day 4. This radiation dose was determined based on previous study [36].

We first investigated the safety and toxicity of lenvatinib and radiation combination therapy in tumorbearing mice. The mice were weighed every other day (Fig. 4b) and the combination therapy had little effect on their body weight. Furthermore, the relative body weights on day 14 in mice administered combination therapy were significantly higher than those of the control mice. Next, we evaluated the toxicity of the combination therapy to the major organs, including the liver, lungs, spleen, and kidneys. Pathological examinations showed no abnormal changes in any of the treated tissues (Fig. 4c).

The tumor dimensions were measured using a caliper every alternate day. The tumor growth curves are shown in Fig. 4d. Compared to the group administered lenvatinib alone, tumor growth at day 14 was significantly inhibited in the group administered combination therapy. However, although the tumor volume in the group administered a combination therapy of sorafenib and radiation was reduced by $22.7 \%$ on day 14 compared to that for sorafenib alone, we observed no significant difference between both treatment groups (Fig. S3).

On day 15 , following the intravenous injection of AuNPs, $\mu$ X-ray CT imaging of the tumors in all groups was performed (Fig. 4e). Tumor volume, vessel volume, and the volume density of the intratumoral vessels were calculated (Fig. 4f). All CT values were significantly decreased in the group treated with combination therapy with lenvatinib and radiation compared to the other three groups. However, we observed no significant differences between the group without treatment (control) and the group treated 6 Gy of radiation alone. These results suggested that lenvatinib promoted radiosensitivity in HCC tumors; 
thus, lenvatinib and radiation combination therapy drastically inhibited tumor growth and intratumoral vasculature.

Moreover, to examine the effects of lenvatinib and radiation combination therapy on the number of microvessels, expansion of the necrotic area, and number of Ki-67-positive cells, pathological analyses were performed using tumor tissues in each treatment group on day 15 (Fig. $5 \mathrm{a}$ ). Ki-67 is a nuclear nonhistone protein present in all active phases of the cell cycle.[37] The results showed that, although there were no significant differences in the numbers of microvessels between the group administered lenvatinib alone and that administered combination therapy, the expansion of the necrotic area and number of Ki-67-positive cells differed significantly between the group administered combination therapy and the other three groups (Fig. 5b-d).

\section{Discussion}

In clinical studies, although lenvatinib treatment reportedly improved the ORR in HCC patients compared to sorafenib treatment [21], the mechanisms for this difference remained unclear. Therefore, it is important to understand how lenvatinib and sorafenib have different effects on tumor vasculature and microenvironment. Our data indicated no clear difference in the volume densities of tumor vessels as measured using $\mu X$-ray CT, between treatment with $3 \mathrm{mg} / \mathrm{kg}$ of lenvatinib and $50 \mathrm{mg} / \mathrm{kg}$ of sorafenib for 4 or 14 days. However, our examination of the effects of treatment with $3 \mathrm{mg} / \mathrm{kg}$ of lenvatinib and 50 $\mathrm{mg} / \mathrm{kg}$ of sorafenib for 4 days via immunostaining with a fluorescent dye and IFP measurement revealed differences in efficacy between the drugs in tumor microvessels and microenvironment. Compared to sorafenib treatment for 4 days, lenvatinib treatment for 4 days reduced the area density of tumor blood vessels (lenvatinib/sorafenib $=0.73$ ) (Fig. 2f) and increased the percentages of vessels covered with pericytes (lenvatinib/sorafenib = 1.31) (Fig. 2g) and perfused vessels (lenvatinib/sorafenib = 1.41) (Fig. 2i), which indicate tumor vessel normalization. With normalization, IFP decreased (lenvatinib/sorafenib $=$ 0.69) (Fig. 3c). The respective lenvatinib/sorafenib values serve as reference data for vascular normalization between the two drugs.

Previously, apatinib, a VEGFR-2 selective inhibitor, was shown to induce vascular normalization [13, 38]. Additionally, sunitinib, a multi-target TKI, also induced the normalization of tumor microvessels and reduced hypoxia in murine squamous cell carcinoma [39]. The results of studies of these angiogenesis inhibitors support the results of lenvatinib in the present study. In contrast, sorafenib did not show the same ability to normalize blood vessels. We showed that sorafenib treatment significantly reduced tumor hypoxia in the treatment group compared to that in the control group (Fig. 3b). Regarding the other findings, a previous study reported that sorafenib prevented the hypoxia-induced expression of hypoxiainducible factor-1alpha (HIF-1a) and HIF-2a, thereby decreasing VEGF expression in neuroblastoma cell lines [40]. These results may support the reduction of tumor hypoxia by sorafenib. In contrast, another study showed that sorafenib treatment reduced microvessels and exacerbated tumor tissue hypoxia in renal cell carcinoma xenografts [41]. Additionally, previous preclinical comparative studies between lenvatinib and sorafenib showed that lenvatinib had more immunomodulatory activities as well as 
enhanced antitumor effects with ICls $[42,43]$. Therefore, the relationship between sorafenib and tissue hypoxia or tumor microenvironment remains unclear and further studies are needed to elucidate this relationship.

The different effects of lenvatinib and sorafenib on tumor vasculature and microenvironment might be caused by the fact that both drugs are multi-target TKIs; thus, the inhibitory activities of each drug against multiple target factors may differ. Lenvatinib has more potent inhibitory activities against VEGFRs and FGFRs than sorafenib, while sorafenib potently inhibited the activities of PDGFRs and RAF [24]. The effects of multi-target TKIs on tumor vessels and the tumor microenvironment might vary with drug dose, treatment duration, and target tumor cell lines.

Tumor vessel normalization improves blood perfusion and reduces hypoxia. Additionally, tumor oxygenation also increases radiosensitivity in tumor tissues [8,35]. In this study, lenvatinib and radiation combination therapy had a marked antitumor effect on HCC tumors. As a result of the enhancing effect of the combination therapy, not only the reduction effect of tumor volume, vessel volume, and volume density of tumor vessels but also the expansion of necrotic area and reduction of Ki-67-positive cells were observed. In contrast, the combination therapy with sorafenib and radiation did not show a remarkable antitumor effect in our study.

A previous preclinical study demonstrated the antitumor effects of a combination therapy comprising 10 $\mathrm{mg} / \mathrm{kg}$ of sorafenib and $6 \mathrm{~Gy}$ of radiation [36]. In the study, human HCC was subcutaneously implanted into the mouse and experiments were performed. Therefore, in tumor-bearing mice, the cancer cells were derived from humans, while the tumor vascular endothelial cells were derived from mice. On the other hand, since we performed experiments using tumor-bearing mice transplanted with mouse HCC cells, the different effects of combination therapy of sorafenib and radiation between previous studies and our study may be due to different experimental conditions.

Among patients with HCC, lenvatinib reportedly showed a greater ORR compared to that in sorafenib [21]. Therefore, lenvatinib and radiation combination therapy could potentiate antitumor effects in clinical practice. However, since preclinical results alone cannot have excessive expectations of combination therapy, further investigations of the effects of combination therapy in preclinical settings and clinical verification in $\mathrm{HCC}$ are needed and careful determination of the indications is important.

In conclusion, imaging data of HCC tumor-bearing mice using $\mu \mathrm{X}$-ray CT with AuNPs and immunohistochemistry showed that lenvatinib treatment induced vascular normalization and improvement of intratumoral microenvironments in HCC tumors earlier and more effectively than sorafenib treatment. Moreover, such changes increased the radiosensitivity of the tumors and enhanced the effect of lenvatinib and radiation combination therapy, suggesting that this combination therapy may be a powerful potential application against HCC.

\section{Declarations}




\section{Fundings:}

A portion of this work was supported by a Grant-in-Aid for Challenging Exploratory Research (19K22549) from the Japan Society for the Promotion of Science (JSPS) (K.G.), a Grant-in-Aid for Scientific Research (C) (19K12749) from the JSPS (N.K. and K.G.), and a Grant-in-Aid for Scientific Research (C) (18K08697) from the JSPS (R.N. and K.G.).

\section{Conflicts of interest:}

All authors declare no commercial or financial conflict of interest.

\section{Ethics approval:}

This paper does not involve human tissues or human data. All animal procedures were reviewed and approved by the Committee on Animal Experiments at Tohoku University.

\section{Consent to participate:}

Not applicable for that section.

\section{Consent for publication:}

Not applicable for that section.

\section{Availability of data and material:}

All data and materials are available in this study.

\section{Code availability:}

Not applicable for that section.

\section{Author's contributions}

Norikazu Une and Kohsuke Gonda conceived and designed the experiments. Norikazu Une, Tomoya Inose and Chihiro Kato performed the experiments. Norikazu Une, Tomoya Inose and Chihiro Kato analyzed the data. Norikazu Une, Mineto Ohta, Tomoya Inose, Chihiro Kato, Ryuichi Nishimura, Hiroshi Tada, Shigehito Miyagi, Takanori Ishida, Michiaki Unno, Takashi Kamei, and Kohsuke Gonda discussed the results. Norikazu Une and Kohsuke Gonda wrote the manuscript. All authors read and approved the final version of the manuscript.

\section{Acknowledgements}

We thank Y. Ishigaki for technical assistance. This work was supported by grants from the Japan Society for the Promotion of Science (JSPS). 


\section{References}

1. Folkman J. Tumor angiogenesis: therapeutic implications. N Engl J Med. 1971;285(21):1182-6. doi:10.1056/nejm197111182852108.

2. Hanahan D, Weinberg RA. Hallmarks of cancer: the next generation. Cell. 2011;144(5):646-74. doi:10.1016/j.cell.2011.02.013.

3. Carmeliet P, Jain RK. Angiogenesis in cancer and other diseases. Nature. 2000;407(6801):249-57. doi:10.1038/35025220.

4. Bergers G, Benjamin LE. Tumorigenesis and the angiogenic switch. Nat Rev Cancer. 2003;3(6):40110. doi:10.1038/nrc1093.

5. Jain RK. Molecular regulation of vessel maturation. Nat Med. 2003;9(6):685-93. doi:10.1038/nm0603-685.

6. Carmeliet P, Jain RK. Molecular mechanisms and clinical applications of angiogenesis. Nature. 2011;473(7347):298-307. doi:10.1038/nature10144.

7. Folkman J. Anti-angiogenesis: new concept for therapy of solid tumors. Ann Surg. 1972;175(3):40916. doi:10.1097/00000658-197203000-00014.

8. Dewhirst MW, Cao Y, Moeller B. Cycling hypoxia and free radicals regulate angiogenesis and radiotherapy response. Nat Rev Cancer. 2008;8(6):425-37. doi:10.1038/nrc2397.

9. Wilson WR, Hay MP. Targeting hypoxia in cancer therapy. Nat Rev Cancer. 2011;11(6):393-410. doi:10.1038/nrc3064.

10. Jain RK. Normalization of tumor vasculature: an emerging concept in antiangiogenic therapy. Science. 2005;307(5706):58-62. doi:10.1126/science.1104819.

11. Shen G, Li Y, Du T, Shi G, Dai L, Chen X, et al. SKLB1002, a novel inhibitor of VEGF receptor 2 signaling, induces vascular normalization to improve systemically administered chemotherapy efficacy. Neoplasma. 2012;59(5):486-93. doi:10.4149/neo_2012_062.

12. Martin JD, Seano G, Jain RK. Normalizing Function of Tumor Vessels: Progress, Opportunities, and Challenges. Annu Rev Physiol. 2019;81:505-34. doi:10.1146/annurev-physiol-020518-114700.

13. Shigeta K, Datta M, Hato T, Kitahara S, Chen IX, Matsui A, et al. Dual Programmed Death Receptor-1 and Vascular Endothelial Growth Factor Receptor-2 Blockade Promotes Vascular Normalization and Enhances Antitumor Immune Responses in Hepatocellular Carcinoma. Hepatology. 2020;71(4):1247-61. doi:10.1002/hep.30889.

14. Finn RS, Qin S, Ikeda M, Galle PR, Ducreux M, Kim TY, et al. Atezolizumab plus Bevacizumab in Unresectable Hepatocellular Carcinoma. N Engl J Med. 2020;382(20):1894-905. doi:10.1056/NEJMoa1915745.

15. Bray F, Ferlay J, Soerjomataram I, Siegel RL, Torre LA, Jemal A. Global cancer statistics 2018: GLOBOCAN estimates of incidence and mortality worldwide for 36 cancers in 185 countries. CA Cancer J Clin. 2018;68(6):394-424. doi:10.3322/caac.21492. 
16. Bruix J, Reig M, Sherman M. Evidence-Based Diagnosis, Staging, and Treatment of Patients With Hepatocellular Carcinoma. Gastroenterology. 2016;150(4):835-53. doi:10.1053/j.gastro.2015.12.041.

17. Lau WY, Leung TW, Lai BS, Liew CT, Ho SK, Yu SC, et al. Preoperative systemic chemoimmunotherapy and sequential resection for unresectable hepatocellular carcinoma. Ann Surg. 2001;233(2):236-41. doi:10.1097/00000658-200102000-00013.

18. EASL Clinical Practice Guidelines. Management of hepatocellular carcinoma. J Hepatol. 2018;69(1):182-236. doi:10.1016/j.jhep.2018.03.019.

19. Llovet JM, Ricci S, Mazzaferro V, Hilgard P, Gane E, Blanc JF, et al. Sorafenib in advanced hepatocellular carcinoma. N Engl J Med. 2008;359(4):378-90. doi:10.1056/NEJMoa0708857.

20. Cheng AL, Kang YK, Chen Z, Tsao CJ, Qin S, Kim JS, et al. Efficacy and safety of sorafenib in patients in the Asia-Pacific region with advanced hepatocellular carcinoma: a phase III randomised, doubleblind, placebo-controlled trial. Lancet Oncol. 2009;10(1):25-34. doi:10.1016/s1470-2045(08)702857.

21. Kudo M, Finn RS, Qin S, Han KH, Ikeda K, Piscaglia F, et al. Lenvatinib versus sorafenib in first-line treatment of patients with unresectable hepatocellular carcinoma: a randomised phase 3 noninferiority trial. Lancet. 2018;391(10126):1163-73. doi:10.1016/s0140-6736(18)30207-1.

22. Bruix J, Qin S, Merle P, Granito A, Huang YH, Bodoky G, et al. Regorafenib for patients with hepatocellular carcinoma who progressed on sorafenib treatment (RESORCE): a randomised, doubleblind, placebo-controlled, phase 3 trial. Lancet. 2017;389(10064):56-66. doi:10.1016/s01406736(16)32453-9.

23. Zhu AX, Kang YK, Yen CJ, Finn RS, Galle PR, Llovet JM, et al. Ramucirumab after sorafenib in patients with advanced hepatocellular carcinoma and increased a-fetoprotein concentrations (REACH-2): a randomised, double-blind, placebo-controlled, phase 3 trial. Lancet Oncol. 2019;20(2):282-96. doi:10.1016/s1470-2045(18)30937-9.

24. Tohyama O, Matsui J, Kodama K, Hata-Sugi N, Kimura T, Okamoto K, et al. Antitumor activity of lenvatinib (e7080): an angiogenesis inhibitor that targets multiple receptor tyrosine kinases in preclinical human thyroid cancer models. J Thyroid Res. 2014;2014:638747. doi:10.1155/2014/638747.

25. Okamoto K, Ikemori-Kawada M, Jestel A, von König K, Funahashi Y, Matsushima T, et al. Distinct binding mode of multikinase inhibitor lenvatinib revealed by biochemical characterization. ACS Med Chem Lett. 2015;6(1):89-94. doi:10.1021/ml500394m.

26. Kim KJ, Li B, Winer J, Armanini M, Gillett N, Phillips HS, et al. Inhibition of vascular endothelial growth factor-induced angiogenesis suppresses tumour growth in vivo. Nature. 1993;362(6423):841-4. doi:10.1038/362841a0.

27. Weidner N, Carroll PR, Flax J, Blumenfeld W, Folkman J. Tumor angiogenesis correlates with metastasis in invasive prostate carcinoma. Am J Pathol. 1993;143(2):401-9. 
28. Sun C, Li J, Wang B, Shangguan J, Figini M, Shang N, et al. Tumor angiogenesis and bone metastasis - Correlation in invasive breast carcinoma. J Immunol Methods. 2018;452:46-52. doi:10.1016/j.jim.2017.10.006.

29. Fidler IJ. Tumor heterogeneity and the biology of cancer invasion and metastasis. Cancer Res. 1978;38(9):2651-60.

30. Lu LC, Hsu CH, Hsu C, Cheng AL. Tumor Heterogeneity in Hepatocellular Carcinoma: Facing the Challenges. Liver Cancer. 2016;5(2):128-38. doi:10.1159/000367754.

31. Nakagawa T, Gonda K, Kamei T, Cong L, Hamada Y, Kitamura N, et al. X-ray computed tomography imaging of a tumor with high sensitivity using gold nanoparticles conjugated to a cancer-specific antibody via polyethylene glycol chains on their surface. Sci Technol Adv Mater. 2016;17(1):387-97. doi:10.1080/14686996.2016.1194167.

32. Fadnes HO, Reed RK, Aukland K. Interstitial fluid pressure in rats measured with a modified wick technique. Microvasc Res. 1977;14(1):27-36. doi:10.1016/0026-2862(77)90138-8.

33. Niwano M, Arii S, Mori A, Ishigami S, Harada T, Mise M, et al. Inhibition of tumor growth and microvascular angiogenesis by the potent angiogenesis inhibitor, TNP-470, in rats. Surg Today. 1998;28(9):915-22. doi:10.1007/s005950050252.

34. Mazzetti S, Frigerio S, Gelati M, Salmaggi A, Vitellaro-Zuccarello L. Lycopersicon esculentum lectin: an effective and versatile endothelial marker of normal and tumoral blood vessels in the central nervous system. Eur J Histochem. 2004;48(4):423-8. doi:10.4081/916.

35. Sørensen BS, Horsman MR. Tumor Hypoxia: Impact on Radiation Therapy and Molecular Pathways. Front Oncol. 2020;10:562. doi:10.3389/fonc.2020.00562.

36. Chen JC, Chuang HY, Hsu FT, Chen YC, Chien YC, Hwang JJ. Sorafenib pretreatment enhances radiotherapy through targeting MEK/ERK/NF-KB pathway in human hepatocellular carcinomabearing mouse model. Oncotarget. 2016;7(51):85450-63. doi:10.18632/oncotarget.13398.

37. Soliman NA, Yussif SM. Ki-67 as a prognostic marker according to breast cancer molecular subtype. Cancer Biol Med. 2016;13(4):496-504. doi:10.20892/j.issn.2095-3941.2016.0066.

38. Zhou K, Zhang JW, Wang QZ, Liu WY, Liu JL, Yao L, et al. Apatinib, a selective VEGFR2 inhibitor, improves the delivery of chemotherapeutic agents to tumors by normalizing tumor vessels in LoVo colon cancer xenograft mice. Acta Pharmacol Sin. 2019;40(4):556-62. doi:10.1038/s41401-0180058-y.

39. Matsumoto S, Batra S, Saito K, Yasui H, Choudhuri R, Gadisetti C, et al. Antiangiogenic agent sunitinib transiently increases tumor oxygenation and suppresses cycling hypoxia. Cancer Res. 2011;71(20):6350-9. doi:10.1158/0008-5472.Can-11-2025.

40. Nilsson MB, Zage PE, Zeng L, Xu L, Cascone T, Wu HK, et al. Multiple receptor tyrosine kinases regulate HIF-1 alpha and HIF-2alpha in normoxia and hypoxia in neuroblastoma: implications for antiangiogenic mechanisms of multikinase inhibitors. Oncogene. 2010;29(20):2938-49. doi:10.1038/onc.2010.60. 
41. Yu W, Zhao S, Zhao Y, Fatema CN, Murakami M, Nishijima Kl, et al. Changes in tumor oxygen state after sorafenib therapy evaluated by (18)F-fluoromisonidazole hypoxia imaging of renal cell carcinoma xenografts. Oncol Lett. 2017;14(2):2341-6. doi:10.3892/ol.2017.6371.

42. Kimura T, Kato Y, Ozawa Y, Kodama K, Ito J, Ichikawa K, et al. Immunomodulatory activity of lenvatinib contributes to antitumor activity in the Hepa1-6 hepatocellular carcinoma model. Cancer Sci. 2018;109(12):3993-4002. doi:10.1111/cas.13806.

43. Deng H, Kan A, Lyu N, Mu L, Han Y, Liu L, et al. Dual Vascular Endothelial Growth Factor Receptor and Fibroblast Growth Factor Receptor Inhibition Elicits Antitumor Immunity and Enhances Programmed Cell Death-1 Checkpoint Blockade in Hepatocellular Carcinoma. Liver Cancer. 2020;9(3):338-57. doi:10.1159/000505695.

\section{Figures}


a

- saline (control)

- $30 \mathrm{mg} / \mathrm{kg}$ or $50 \mathrm{mg} / \mathrm{kg}$ of sorafenib

- $3 \mathrm{mg} / \mathrm{kg}$ or $10 \mathrm{mg} / \mathrm{kg}$ of lenvatinib

once daily, oral gavage

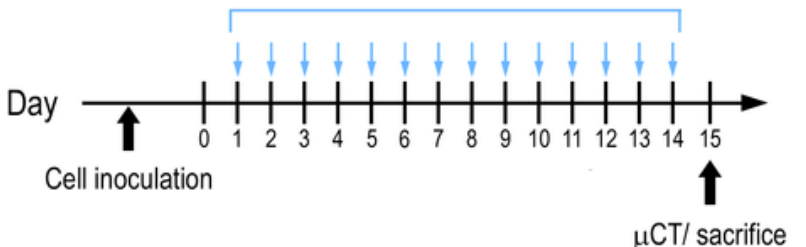

C

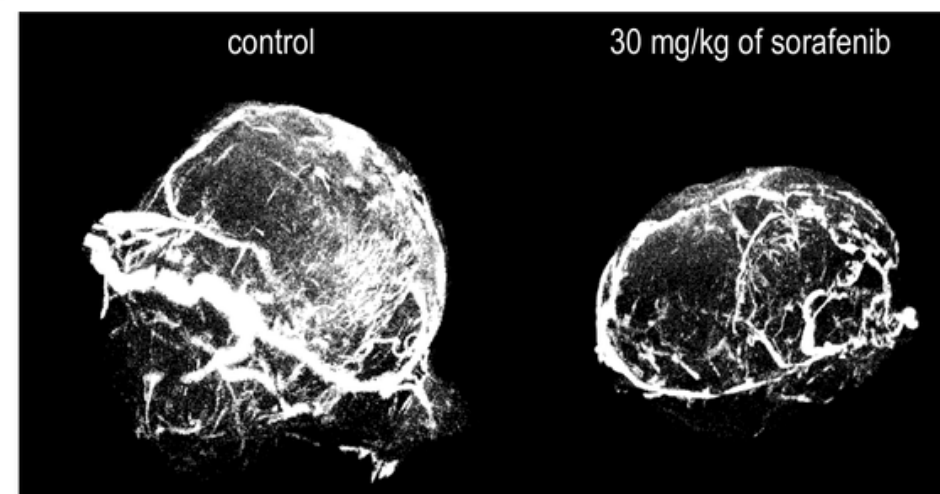

d
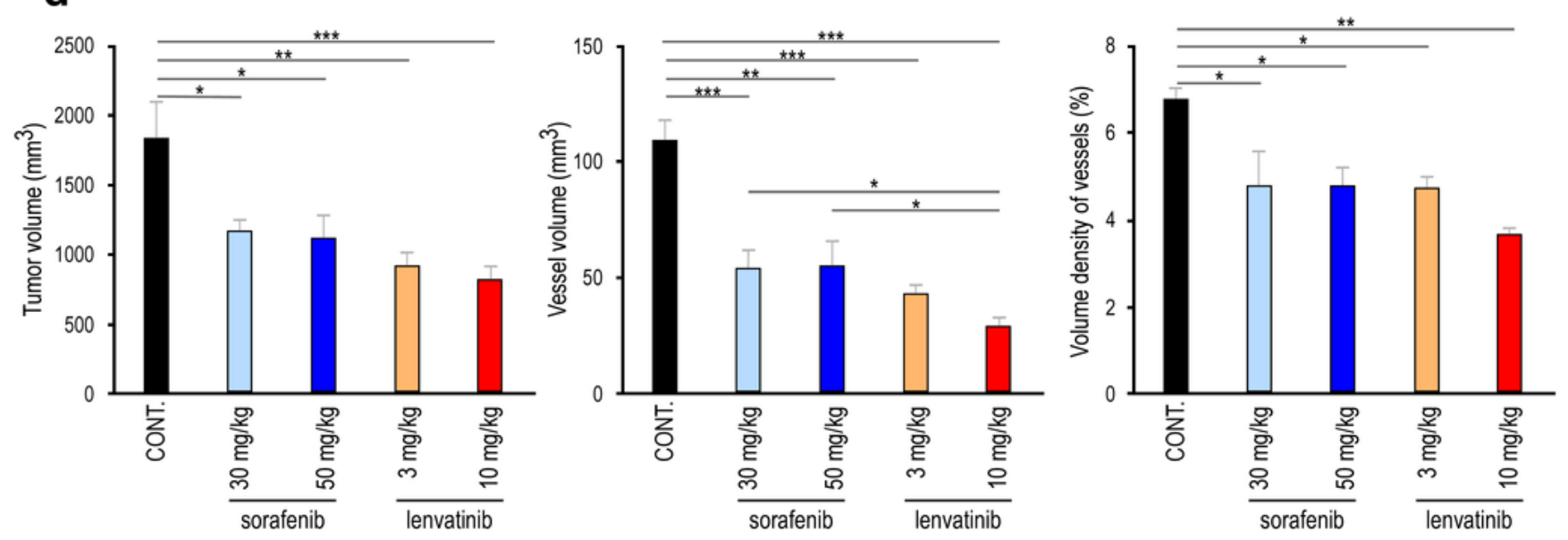

\section{Figure 1}

Inhibitory effects of lenvatinib and sorafenib treatment for 14 days on tumor growth and tumor vessel density. (a) Schematic representation of the 14-day sorafenib and lenvatinib treatment protocols. Tumorbearing mice transplanted with Hepa1-6 cells were randomly assigned into five groups administered saline (Control) ( $\mathrm{n}=4$ ), $50 \mathrm{mg} / \mathrm{kg}$ or $30 \mathrm{mg} / \mathrm{kg}$ of sorafenib ( $\mathrm{n}=5$ per group), and $10 \mathrm{mg} / \mathrm{kg}$ or $3 \mathrm{mg} / \mathrm{kg}$ of lenvatinib ( $\mathrm{n}=5$ per group). From day 1 , saline, lenvatinib, or sorafenib were administered by oral 
gavage for 14 consecutive days. On day 15 , we imaged the tumor vessels by $\mu$ X-ray CT with AuNPs as contrast agents. (b) Growth curves of the groups treated with saline, sorafenib, or lenvatinib. The tumor dimensions were measured using a caliper. Sorafenib and lenvatinib treatment showed significantly delayed tumor growth on day 14 compared to that in the control group. (c) In vivo 3D $\mu X$-ray CT imaging of the tumor vessels. AuNP contrast agents were intravenously injected into mice tail veins and the tumor vessels were imaged by $\mu \mathrm{X}$-ray CT on day 15 . Representative images of the tumor vessels are shown. (d) Quantification of in vivo $\mu$ X-ray CT imaging of tumor vessels treated with saline, sorafenib, or lenvatinib. Tumor volume, tumor vessel volume, and volume density of vessels calculated by $\mu X$-ray CT imaging are shown ( $n=4$ per group). The tumor volumes, vessel volumes, and vessel volume densities of those treated with sorafenib and lenvatinib were significantly lower than those in the control group. Additionally, the vessel volume in the $10 \mathrm{mg} / \mathrm{kg}$ lenvatinib group was significantly decreased compared to those in the $30 \mathrm{mg} / \mathrm{kg}$ and $50 \mathrm{mg} / \mathrm{kg}$ sorafenib groups ( $10 \mathrm{mg} / \mathrm{kg}$ lenvatinib, $27.9 \pm 3.71 \mathrm{~mm} 3 ; 30 \mathrm{mg} / \mathrm{kg}$ of sorafenib, $53.0 \pm 8.37 \mathrm{~mm} 3 ; 50 \mathrm{mg} / \mathrm{kg}$ of sorafenib, $54.6 \pm 9.98 \mathrm{~mm} 3)$. The data represent the mean \pm SEM. ${ }^{*} p<0.05 ;{ }^{* *} p<0.01 ; * \star * p<0.001$. 
a

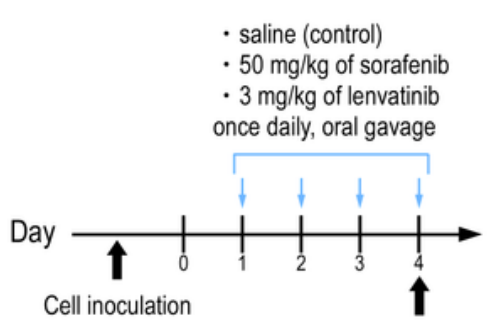

Tumor analysis

(1) AuNPs iv $\rightarrow \mu \mathrm{CT}$

(2) Lectin perfusion

(3) IFP measurement b

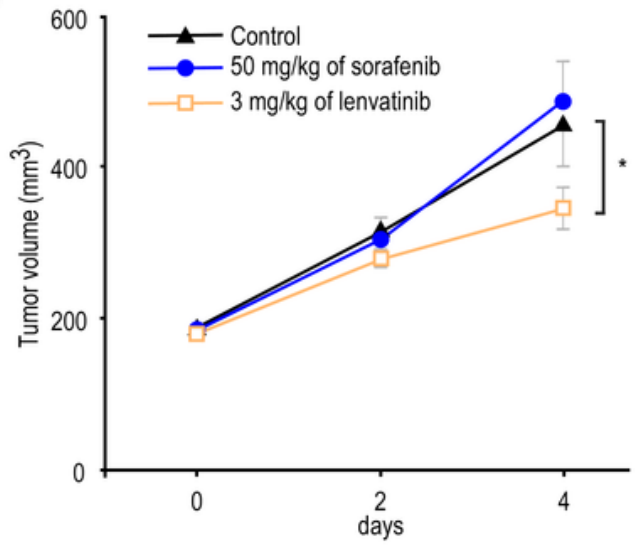

d

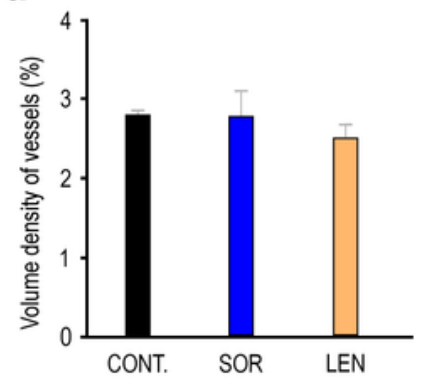

f
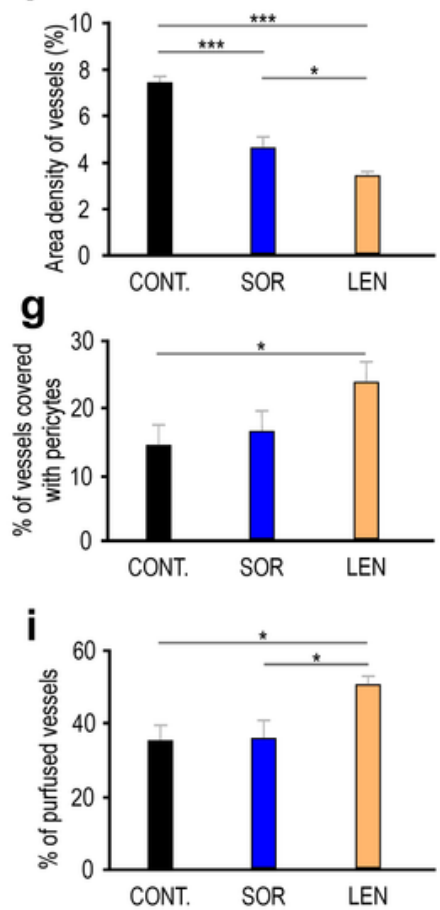

Figure 2

Effects of 4-day sorafenib and lenvatinib treatment on tumor vasculature. (a) Schematic representation of the 4-day sorafenib and lenvatinib treatment protocols. Tumor-bearing mice transplanted with Hepa1-6 cells were randomly assigned into three groups administered saline (Control), $50 \mathrm{mg} / \mathrm{kg}$ sorafenib, and 3 $\mathrm{mg} / \mathrm{kg}$ lenvatinib. On day 4, we imaged and analyzed tumor vessels by $\mu \mathrm{X}$-ray $\mathrm{CT}$ and microvessel vasculature and function by immunohistochemistry; we then measured IFP and the degree of hypoxic 
area in the tumors. (b) Tumor growth curves following treatment with sorafenib and lenvatinib for 4 days ( $n=6$ per group). (c) Representative 3D tumor vessel images obtained by $\mu$ X-ray CT in tumors treated with saline, sorafenib, or lenvatinib. (d) Analyses of $\mu$ X-ray CT imaging for vessel density of intratumor vessels after saline, sorafenib, or lenvatinib treatment. No significant differences were observed among the three groups ( $n=3$ per group). (e) The morphology and maturity changes in the microvessels of tumors treated with saline, sorafenib, or lenvatinib. Endothelial cells and pericytes were stained with antiCD31 (green) and anti-a- smooth muscle actin (SMA) (red) antibodies, respectively. Representative images are shown. Scale bar, $50 \mu \mathrm{m}$. (f) Quantification of the area densities of microvessels in tumor tissues treated with saline, sorafenib, or lenvatinib. Lenvatinib treatment significantly reduced the area densities of tumor microvessels compared to control and sorafenib treatments. (3.43 $\pm 0.19 \%$ vs. $7.47 \pm$ $0.26 \%$ vs. $4.71 \pm 0.37 \%$, respectively, $n=3$ per group). (g) Pericyte coverage of vessels in tumor tissues treated with saline, sorafenib, or lenvatinib and sorafenib. The percentage of vessels covered with pericytes in tumor tissues treated with lenvatinib was significantly higher than those in vessels receiving control treatments ( $24.2 \pm 2.82 \%$ vs. $14.7 \pm 2.77 \%$, respectively, $n=3$ per group). (h) Identification of functional tumor vessels treated with saline, sorafenib, or lenvatinib. All tumor microvessels were stained with anti-CD31 antibody (green) and lectin-labeled perfused vessels are shown in red. Scale bar, $50 \mu \mathrm{m}$. (i) Quantification of perfused vessels in tumor tissues. The percentage of perfused vessels in tumors treated with lenvatinib were significantly higher than those in vessels receiving control and sorafenib treatments $(50.6 \pm 2.32 \%$ vs. $35.5 \pm 3.96 \%$ vs. $35.9 \pm 5.23 \%$, respectively, $n=3$ per group). Data represent the mean \pm SEM. ${ }^{*} p<0.05 ;{ }^{* *} p<0.01 ;{ }^{* \star *} p<0.001$. In (d), (f), (g), and (i), CONT., SOR, and LEN indicate control, sorafenib, and lenvatinib, respectively.
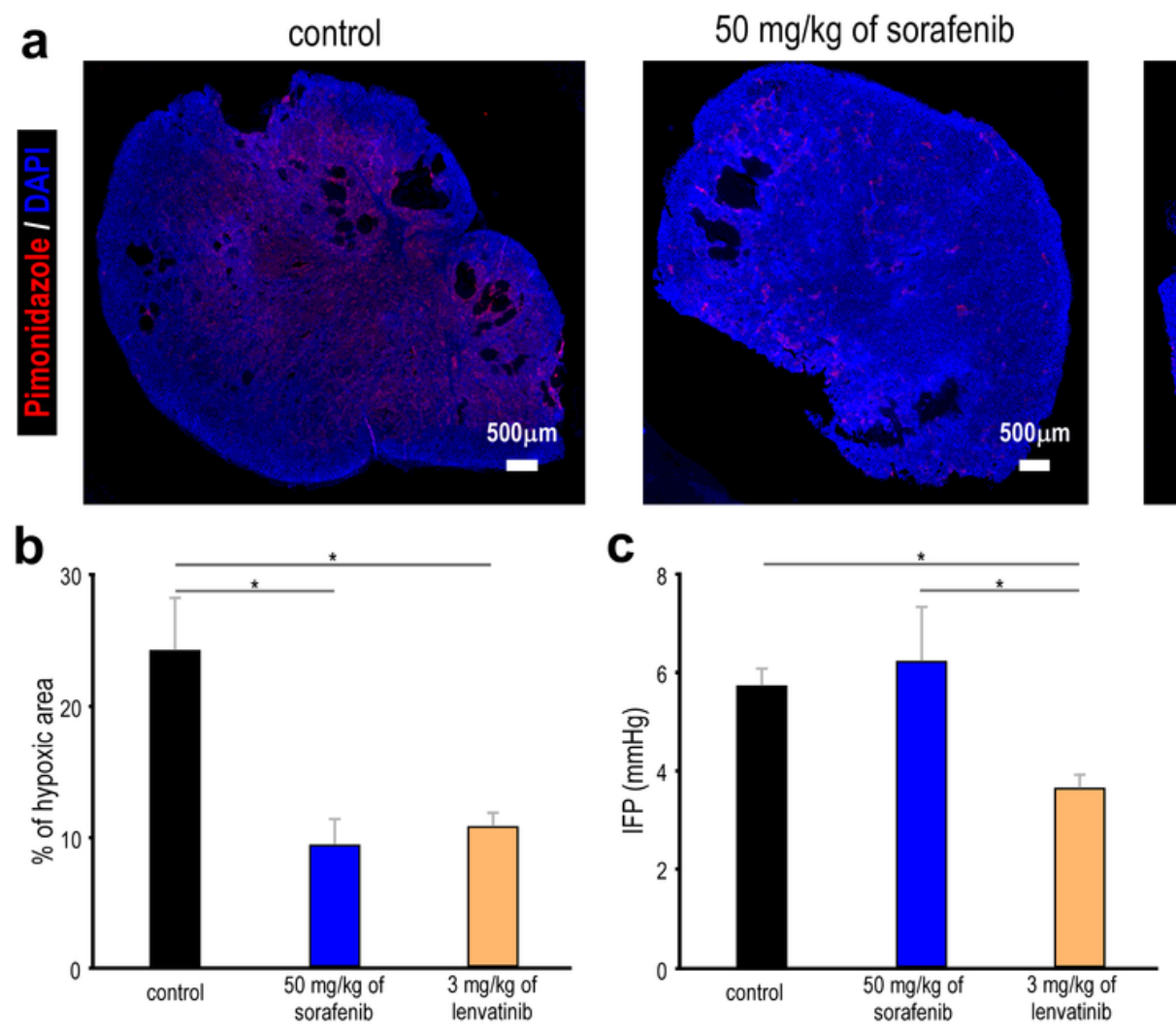

$3 \mathrm{mg} / \mathrm{kg}$ of lenvatinib

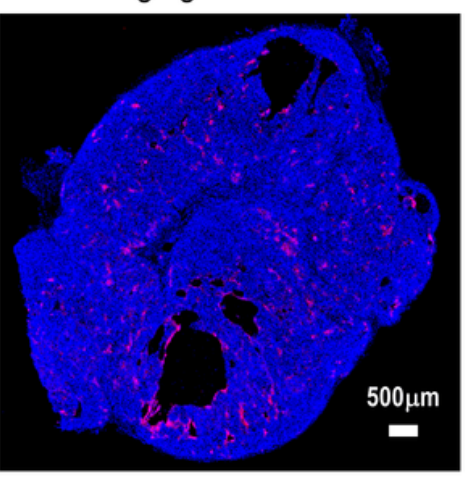

Figure 3 
Microenvironments of tumors treated with sorafenib and lenvatinib. (a) Identification of hypoxic areas in tumor tissues. Tumor tissue sections were double-stained with anti-pimonidazole antibody and DAPI and then evaluated. Hypoxic areas are shown in red. Scale bar, $500 \mu \mathrm{m}$. Blue represents DAPI staining. (b) Quantification of tissue hypoxia. The percentage of hypoxic area was calculated as the pimonidazolepositive area per viable area (DAPI). Sorafenib and lenvatinib treatment showed significantly reduced hypoxic areas compared to that in the control ( $n=5$ per group). (c) Measurement of IFP in the tumors. IFP was measured with the wick-in-needle technique. The IFP in tumors treated with lenvatinib was significantly lower than that with tumors treated with saline (control) and sorafenib $(3.65 \pm 0.26 \mathrm{mmHg}$ vs. $5.71 \pm 0.38 \mathrm{mmHg}$ vs. $6.23 \pm 1.06 \mathrm{mmHg}$; respectively, $\mathrm{n}=5$ per group). Data represent the mean \pm SEM. ${ }^{*} p<0.05$. 
a

irradiation (6Gy)

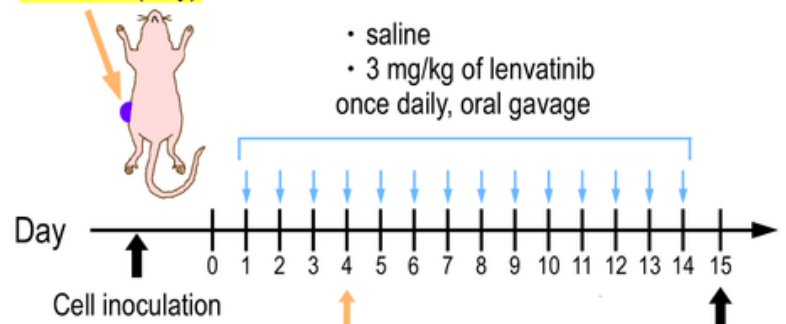

with/without irradiation (6Gy)

C
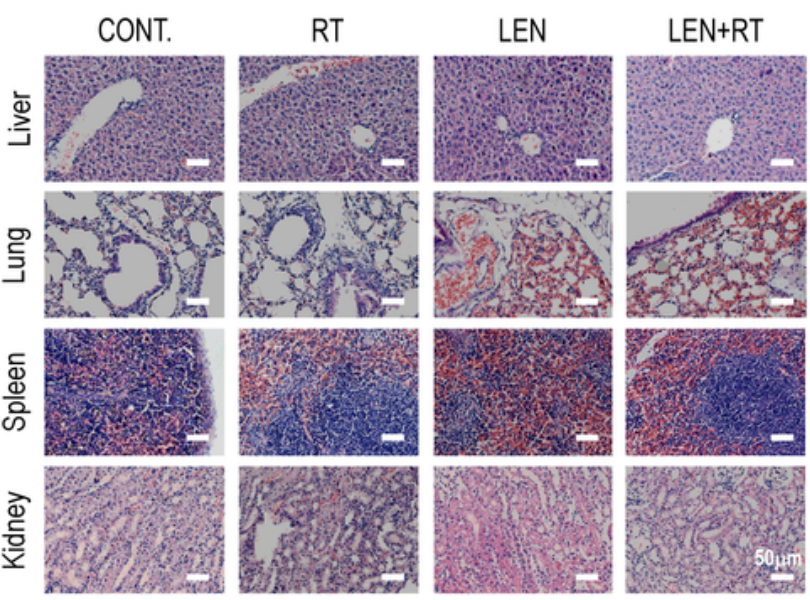

b

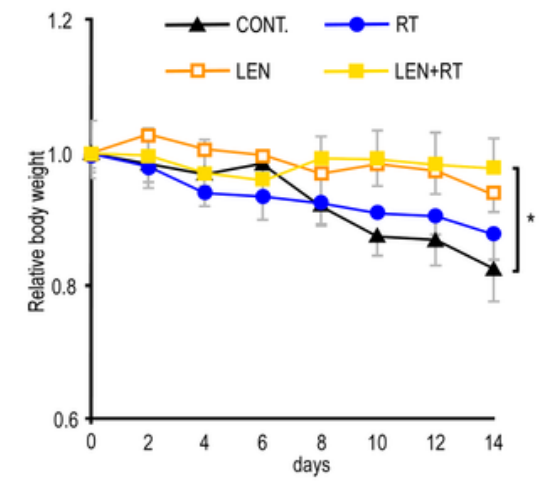

d

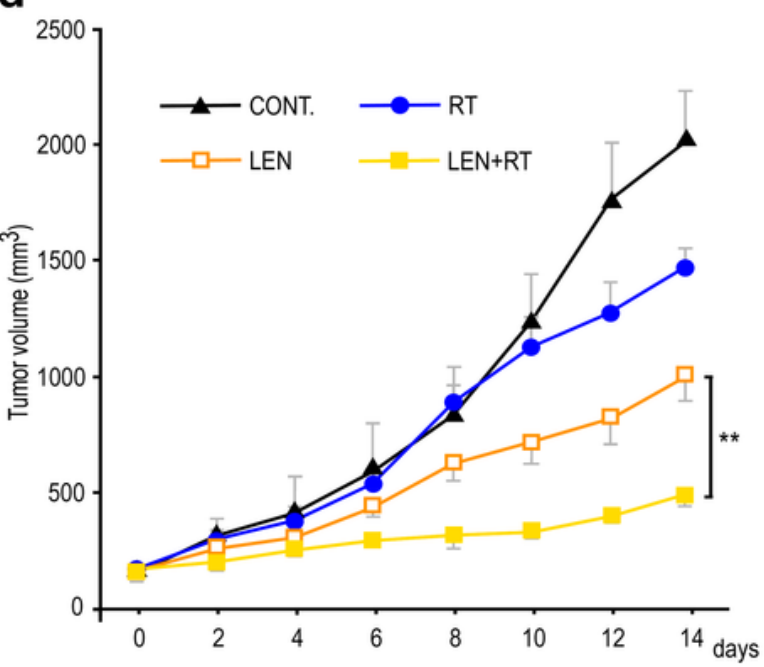

e

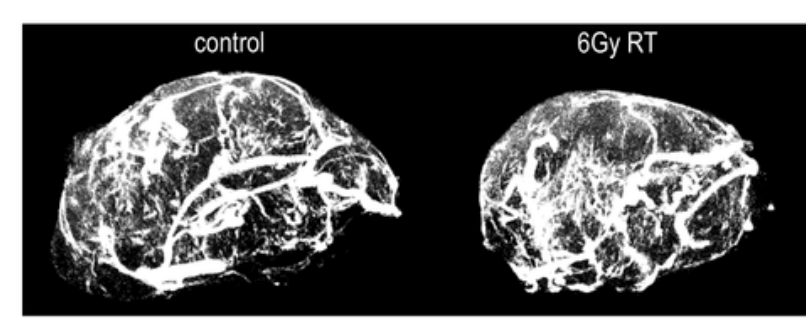

$3 \mathrm{mg} / \mathrm{kg}$ of lenvatinib

lenvatinib + RT
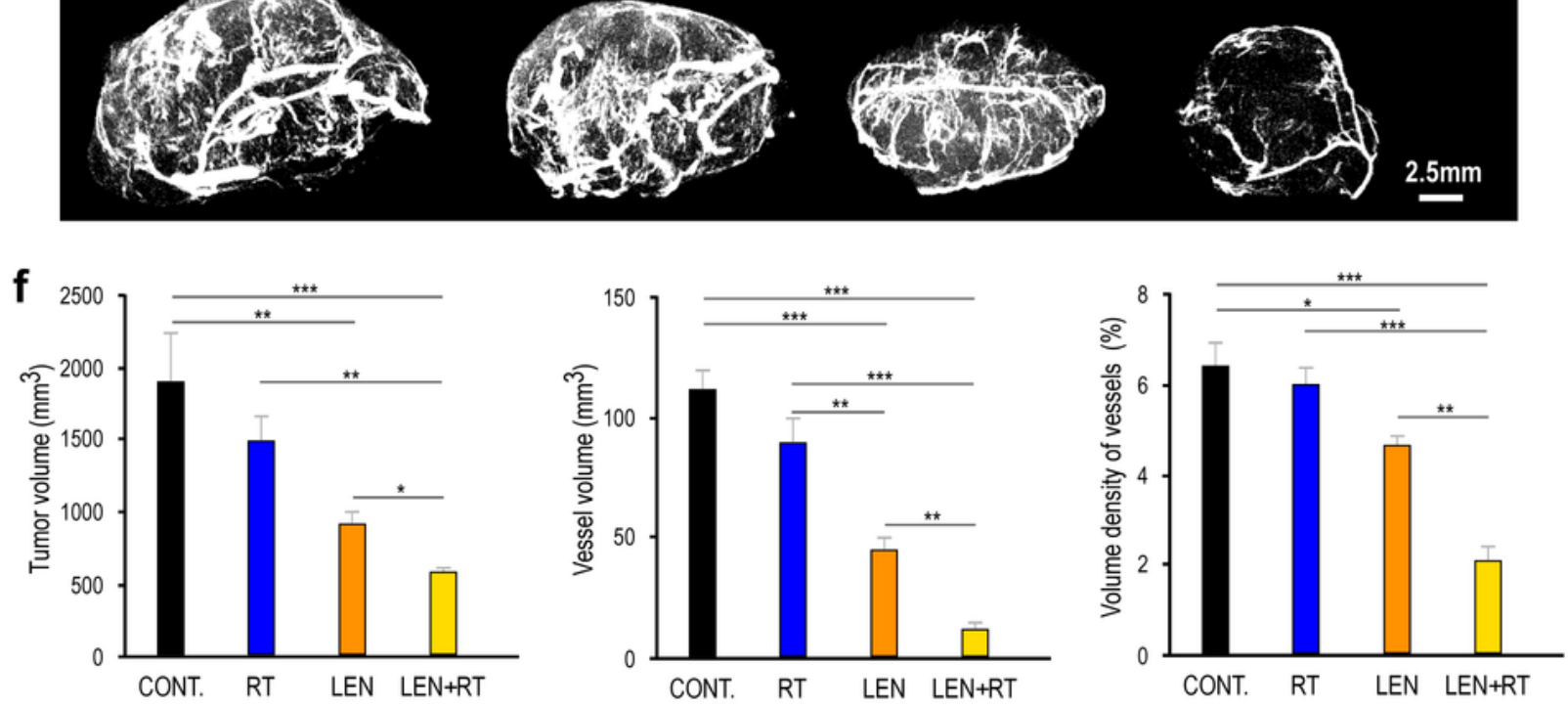

Figure 4

Effect of lenvatinib and radiation combination therapy on HCC tumors. (a) Schematic representation of the 14-day treatment protocol using lenvatinib and radiation combination therapy. Tumor-bearing mice were treated with saline or $3 \mathrm{mg} / \mathrm{kg}$ lenvatinib for 14 days. On day 4, the tumors were irradiated with or without $6 \mathrm{~Gy}$ of X-rays. The tumor volumes were measured every other day and the mice were scanned by $\mu \mathrm{X}$-ray CT on day 15 to evaluate the tumor vessels. (b) The relative body weights of the mice were 
measured every other day. (c) Representative hematoxylin and eosin (H\&E)-stained images of various organs treated with lenvatinib and irradiation. Pathological examination revealed no abnormal changes in any of the treated tissues. (d) Tumor growth curves of lenvatinib and radiation combination therapy. The tumor volume following treatment with both lenvatinib and radiation was significantly lower than those with lenvatinib alone (474.7 $\pm 10.7 \mathrm{~mm} 3 \mathrm{vs}$. $995.6 \pm 97.1 \mathrm{~mm} 3$, respectively, $\mathrm{n}=5$ per group). (e) In vivo 3D $\mu \mathrm{X}$-ray CT imaging of tumor vessels. Representative images of the tumor vessels are shown. (f) Quantification of $\mu X$-ray CT imaging of intratumor vessels treated with saline, lenvatinib, radiation, or both. Tumor volume, vessel volume, and volume density of vessels treated with the combination of lenvatinib and radiation were significantly lower than those in the other three groups, with significant differences for all except for the comparison between control treatment and $6 \mathrm{~Gy}$ of radiation alone $(\mathrm{n}=4$ per group). Data represent the mean \pm SEM. ${ }^{*} p<0.05 ; * \star p<0.01 ; * \star \star p<0.001$. In (f), CONT., LEN, RAD, and LEN + RAD indicate control, lenvatinib, radiotherapy, and both lenvatinib and radiotherapy, respectively.

a

$\mathrm{CD} 31$

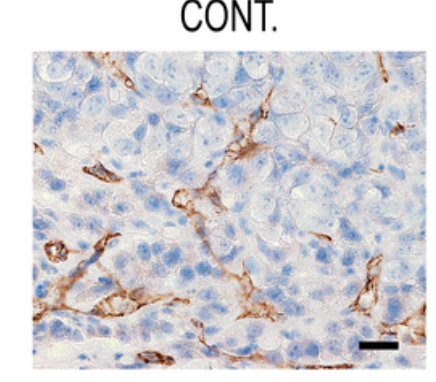

$H \& E$
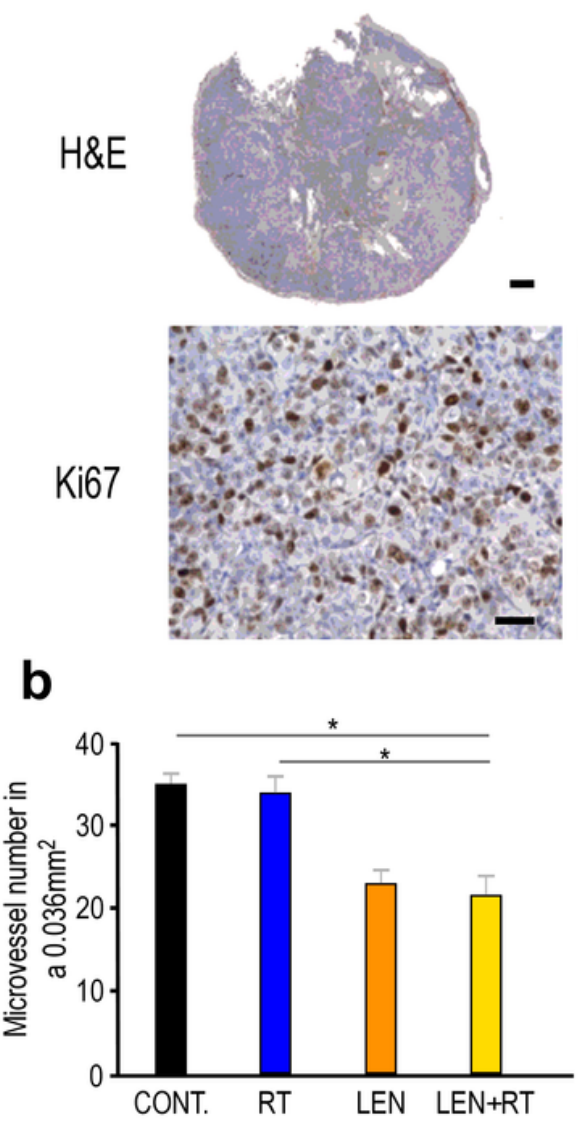

RT
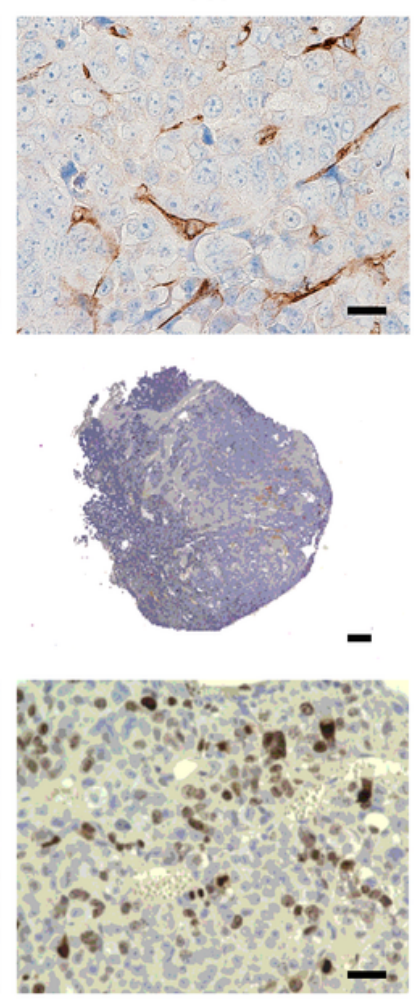

C

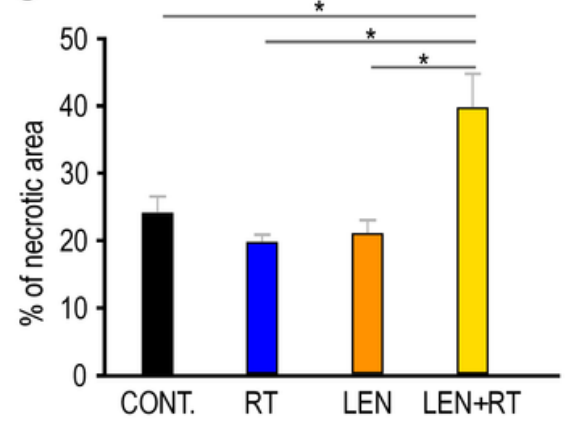

LEN
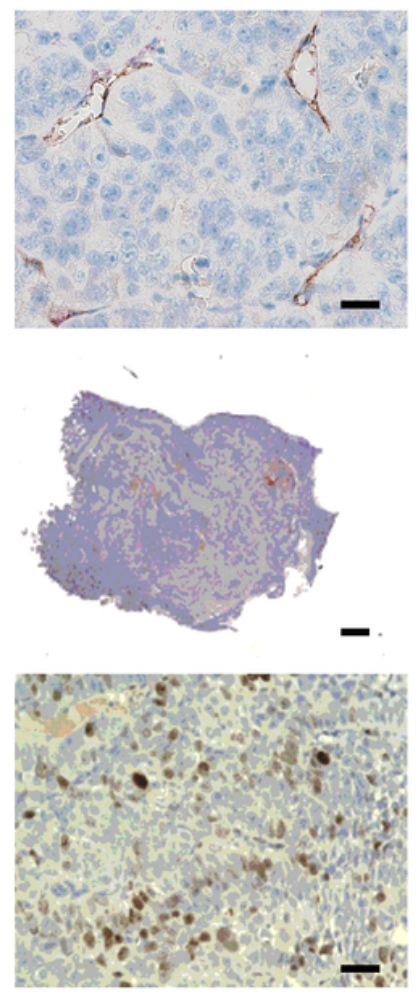

d

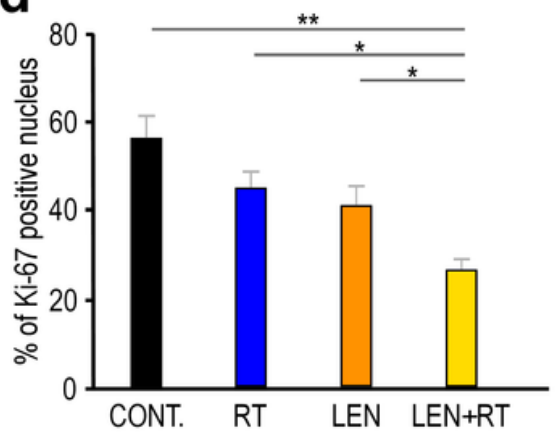

Figure 5 
Effects of lenvatinib and radiation combination therapy on the number of tumor vessels and cell proliferations. (a) Representative images of tumor microvessels stained with anti-CD31 antibody, necrotic areas stained with hematoxylin and eosin (H\&E), and cell proliferation stained with anti-Ki-67 antibody. (b) Quantification of microvessel numbers in $0.036 \mathrm{~mm} 2$ of tumor tissue. The microvessels were stained with anti-CD31 antibody and the numbers of microvessels were counted $(n=5$ tissues from different tumors per group). (c) Quantification of necrotic areas in tumor tissues. The necrotic areas were evaluated based on hematoxylin-positive areas per whole tissue sections. Lenvatinib and radiation combination therapy showed an increased necrotic area $(n=5$ from different tumors per group). (d) Quantification of Ki-67-positive nuclei in tumor tissues. The numbers of Ki-67-positive cells were evaluated. The percentage of Ki-67-positive nuclei was significantly lower in the tumors administered combination therapy ( $n=5$ from different tumors per group). The data represent the mean \pm SEM. ${ }^{*} p<$ $0.05 ;{ }^{*} \mathrm{p}<0.01$. CONT, LEN, RAD, and LEN + RAD indicate control, lenvatinib, radiotherapy, and both lenvatinib and radiotherapy, respectively.

\section{Supplementary Files}

This is a list of supplementary files associated with this preprint. Click to download.

- supplementaryinformation.pdf 